\title{
Universiteit
}

Leiden

The Netherlands

\section{Experimental demonstration of fractional orbital angular momentum entanglement of two photons}

Oemrawsingh, S.S.R.; Ma, X.; Voigt, D.; Aiello, A.; Eliel, E.R.; Hooft, G.W. 't; Woerdman, J.P.

\section{Citation}

Oemrawsingh, S. S. R., Ma, X., Voigt, D., Aiello, A., Eliel, E. R., Hooft, G. W. 't, \& Woerdman, J. P. (2005). Experimental demonstration of fractional orbital angular momentum entanglement of two photons. Physical Review Letters, 95(24), 240501. doi:10.1103/PhysRevLett.95.240501

Version: $\quad$ Not Applicable (or Unknown)

License: $\quad$ Leiden University Non-exclusive license

Downloaded from: https://hdl.handle.net/1887/64320

Note: To cite this publication please use the final published version (if applicable). 


\title{
Experimental Demonstration of Fractional Orbital Angular Momentum Entanglement of Two Photons
}

\author{
S. S. R. Oemrawsingh, ${ }^{*}$ X. Ma, D. Voigt, A. Aiello, E. R. Eliel, G. W. 't Hooft, ${ }^{\dagger}$ and J. P. Woerdman \\ Huygens Laboratory, Leiden University, Post Office Box 9504, 2300 RA Leiden, The Netherlands \\ (Received 29 April 2005; published 8 December 2005)
}

\begin{abstract}
The singular nature of a noninteger spiral phase plate allows easy manipulation of spatial degrees of freedom of photon states. Using two such devices, we have observed very high-dimensional spatial entanglement of twin photons generated by spontaneous parametric down-conversion.
\end{abstract}

DOI: 10.1103/PhysRevLett.95.240501

PACS numbers: 03.67.Mn, 42.50.Dv, 42.65.Pc

In the early 1990s, it was realized that the quantum state of a photon in a Laguerre-Gaussian laser mode $\mathrm{LG}_{p}^{l}$ is an eigenstate of the orbital angular momentum (OAM) operator, with eigenvalue $l \hbar$ ( $l$ integer) [1,2]. The mode functions $\mathrm{LG}_{p}^{l}(l=-\infty \ldots \infty, p=0 \ldots \infty)$ can be used as a basis of an infinite-dimensional Hilbert space, and thus to describe, within the paraxial approximation, a photon state represented by an arbitrary spatial wave function.

According to theory, the twin photons created in an ideal spontaneous parametric down-conversion (SPDC) process are entangled in the full, infinite-dimensional (spatial) Hilbert space; in fact, they are maximally entangled [3,4]. Several years ago, Mair et al. [5] demonstrated OAM entanglement in a 3D subspace of that Hilbert space, in an experiment where they used integer-OAM analyzers. Establishing even higher dimensional entanglement in a realistic SPDC setup is theoretically possible [6], and has been experimentally achieved up to a dimensionality of 6 per photon [7]. It has been recently proposed that spatial entanglement can be demonstrated in an infinite-dimensional $\left(D^{2}=\infty\right)$ subspace [8]. This can be achieved by using fractional-OAM analyzers in view of the fact that fractional-OAM states are coherent superpositions of an infinite number of $\mathrm{LG}_{p}^{l}$ states with integer OAM. Here we present the results of an experiment on fractional-OAM entanglement; apart from confirming the entanglement, we quantify the dimensionality of the photons emitted by the crystal as $D>3700$ per photon, the entangled space having a dimensionality $D^{2}$.

The key element in our approach is the use of a spiral phase plate (SPP) [9,10], shown in Fig. 1, as part of our analyzer. This plate imprints an azimuth-dependent phase retardation on an incident field. The difference in phase retardation $\Delta \phi$ at azimuthal angles 0 and $2 \pi$ is equal to $\Delta \phi \equiv 2 \pi \ell$, whereby the plate adds an average OAM of $\ell \hbar$ per photon. By adjusting the SPP parameters (see the caption of Fig. 1), the SPP index $\ell$ can be tuned to any desired value. If $\ell$ is noninteger, the output field is not cylindrically symmetric [cf. Figure 3(b)] and carries a mixed screw-edge dislocation in the near field, i.e., a singularity with a transverse component; this dislocation is associated with the radial edge of the SPP [10]. Thus, for all noninteger $\ell$, the SPP is characterized by two parameters, namely, the step index $\ell$ itself and the orientation of the radial edge of the SPP, $\alpha$. The latter is varied by rotating the SPP around the beam axis.

Our approach involves the use of two fractional-OAM analyzers (one per arm) in a SPDC setup. Such an analyzer consists of a noninteger SPP, a single-mode fiber, and a detector; the orientations of the radial edges of the SPPs are varied [see Fig. 3(a)]. Because the SPPs have a noninteger step index $\ell$, the fractional-OAM states provide a natural basis to describe the two-photon state [8]. Each element of this fractional basis can be written as an $\ell$ and $\alpha$ dependent superposition of an infinite number of $\mathrm{LG}_{p}^{l}$ states, extending over both $l$ and $p$. Because this superposition changes when we reorient the SPP, we explore an infinitedimensional subspace of the full, (spatial) Hilbert space by systematically varying $\alpha$ (at constant step index $\ell$ ). We stress that the contribution of $\mathrm{LG}_{p}^{l}$ modes with $p \neq 0$ to this superposition is significant; this is caused by the fact that the dislocation imprinted by the SPP on the incident field has a mixed screw-edge character [10].

The most convenient implementation of this scheme is by choosing the noninteger part $\mathcal{L}$ of the step index $\ell$ to be half-integer, i.e., $\mathcal{L}=\frac{1}{2}$; the states with $\alpha=0$ and $\alpha=\pi$ are then orthogonal (independent of the integer part of $\ell$ ) [8]. When we place an analyzer containing an SPP with index $\ell$ in the signal path of a SPDC setup, and one containing an SPP with index $-\ell$ in the idler path, the

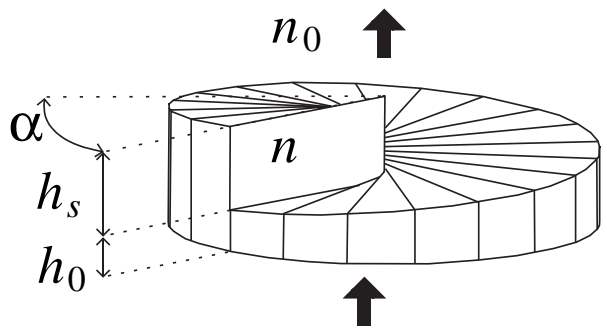

FIG. 1. Schematic drawing of a spiral phase plate with a step index $\ell=h_{s}\left(n-n_{0}\right) / \lambda$, where $h_{s}$ is the step height, $n$ and $n_{0}$ are the refractive indices of the SPP and the surrounding medium, respectively, and $\lambda$ is the wavelength of the incident light. 
coincidence probability as a function of the orientations of the two SPPs is predicted to be [8]

$$
P_{c}\left(\alpha_{s}, \alpha_{i}\right)=|C|^{2}\left(1-\frac{\left|\alpha_{s}-\alpha_{i}\right|}{\pi}\right)^{2}
$$

viz., a parabolic coincidence fringe with a visibility of unity. The constant $C$ depends on the overlap amplitude of the radial part of the two-photon state as emanating from the SPDC crystal, with the radial part of the fiber mode [8]. This parabolic coincidence fringe, which is a function of only the relative orientation of the two SPPs, reveals the entanglement of the twin photons.

The key to success in the experiment lies in the analyzers or, more specifically, in the two SPPs. Our SPPs have been manufactured using a polymer molding technique starting from a micro-machined mold, yielding identical plates with $\ell= \pm 3.48 \pm 0.02$ at the (degenerate SPDC) wavelength of $813 \mathrm{~nm}$. The step of each SPP is not abrupt but, in fact, a linear ramp with an azimuthal width of $6^{\circ}$. Furthermore, the plates show a central "anomaly" with a diameter of about $300 \mu \mathrm{m}$ [11]. The rest of the plate has an rms deviation of $\approx 15 \mathrm{~nm}$ from the ideal shape [12].

The high-dimensional nature of the $\ell=3.5$ SPP is illustrated in Fig. 2(a), which shows a modal decomposition for mode order $\leq 7$ if the input is a fundamental Gaussian mode $\mathrm{LG}_{0}^{0}$ (i.e., the fiber mode). The fidelity of this truncated decomposition is only 43\% [Fig. 2(b)]. When truncating at mode order 20 (corresponding to 231 LG modes), the fidelity becomes $71 \%$. Extrapolation shows that a fidelity of $98 \%$ requires about 125000 LG modes. We have checked that these results remain essentially unchanged when using the actual topography of our
SPP plates; in particular, the finite azimuthal width of the step has no significant effect.

The SPDC setup is shown in Fig. 3(a). The pump laser is a $50 \mathrm{~mW}$ Krypton-ion laser (Spectra Physics 2060) operating at a wavelength of $406.7 \mathrm{~nm}$. Its output is weakly focused on a nonlinear crystal (BBO), with beam waist $w_{0}=0.78 \pm 0.01 \mathrm{~mm}$. The crystal has a thickness $L=$ $1.0 \mathrm{~mm}$ and has been cut for nearly collinear type-II SPDC. Before inserting a fractional-OAM analyzer in each arm, we test the setup by observing polarization entanglement; we achieve $95 \%$ and $92 \%$ fringe visibility in the crystal $(H, V)$ and $45^{\circ}$ basis, respectively [13].

We fix the polarizers in the crystal basis and insert a fractional-OAM analyzer with SPP index $\ell_{s}=+3.48$ in the signal path and an analyzer with SPP index $\ell_{i}=-3.48$ in the idler path. The SPDC cone-crossings in the far field are found to be much larger than the far-field intensity pattern of the analyzer mode, as illustrated in Fig. 3(b). The SPDC intensity varies about $10 \%$ over the SPP diffraction profile.

The SPPs have to be aligned with great precision. In particular, both the rotation axis and the center of each SPP have to be aligned, with a precision of order $1 \mu \mathrm{m}$, with the core of the corresponding single-mode fiber. Only when this amount of care is invested in aligning the optical elements, can one rotate both SPPs to make use of the $\alpha_{s}$ and $\alpha_{i}$ degrees of freedom without losing any signal.

Our experimental results are shown in Fig. 4: four coincidence fringes (obtained for idler SPP-settings $\alpha_{i}=$ $0, \pi / 2, \pi, 3 \pi / 2$ ) as a function of the signal SPP-setting $\alpha_{s}$. These fringes are shown together with the expected coincidence fringes as predicted by Eq. (1). The data show that the coincidence-count rate as a function of the orientation of the signal SPP has a parabola-like shape. Additionally, when the idler SPP is reoriented, we see that the fringe (a)

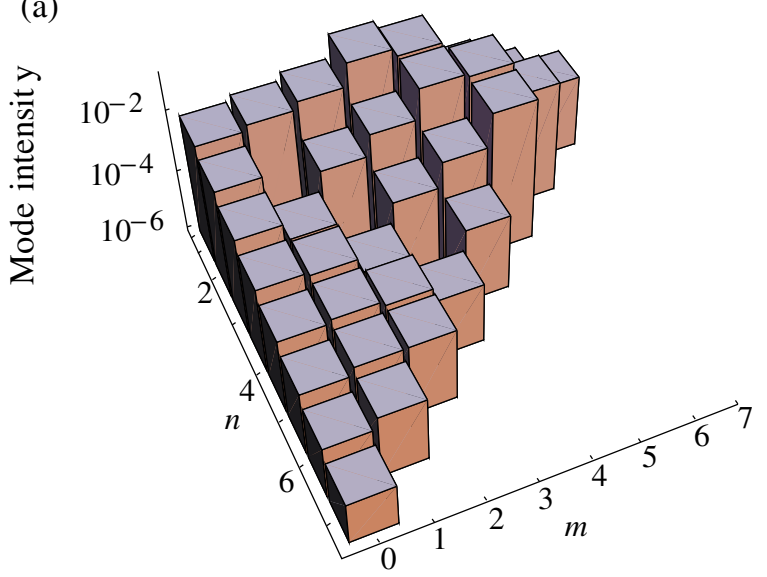

(b)

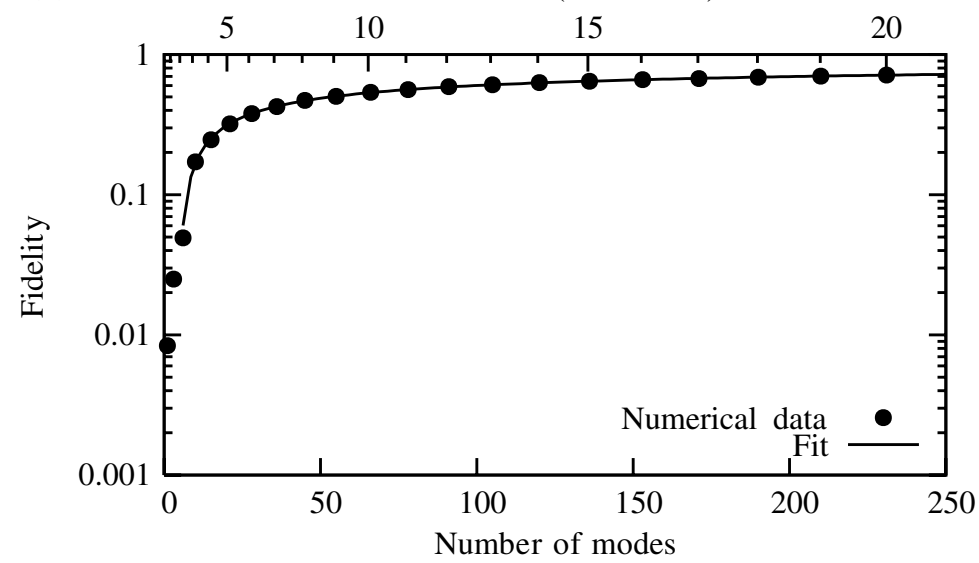

FIG. 2 (color online). Decomposition of the analyzer mode, associated with an SPP with $\ell=3.5$ and a single-mode fiber, into LG modes. (a) The contribution per LG mode for mode orders $m+n \leq 7$, where $l=n-m$ and $p=\min (n, m)$. (b) The fidelity of the mode decomposition as a function of number of modes used in the decomposition. Each of the data points is calculated for LG modes of mode order up to and including the value indicated on the top axis. The solid line is an accurate fit to the numerical data, allowing extrapolation. 


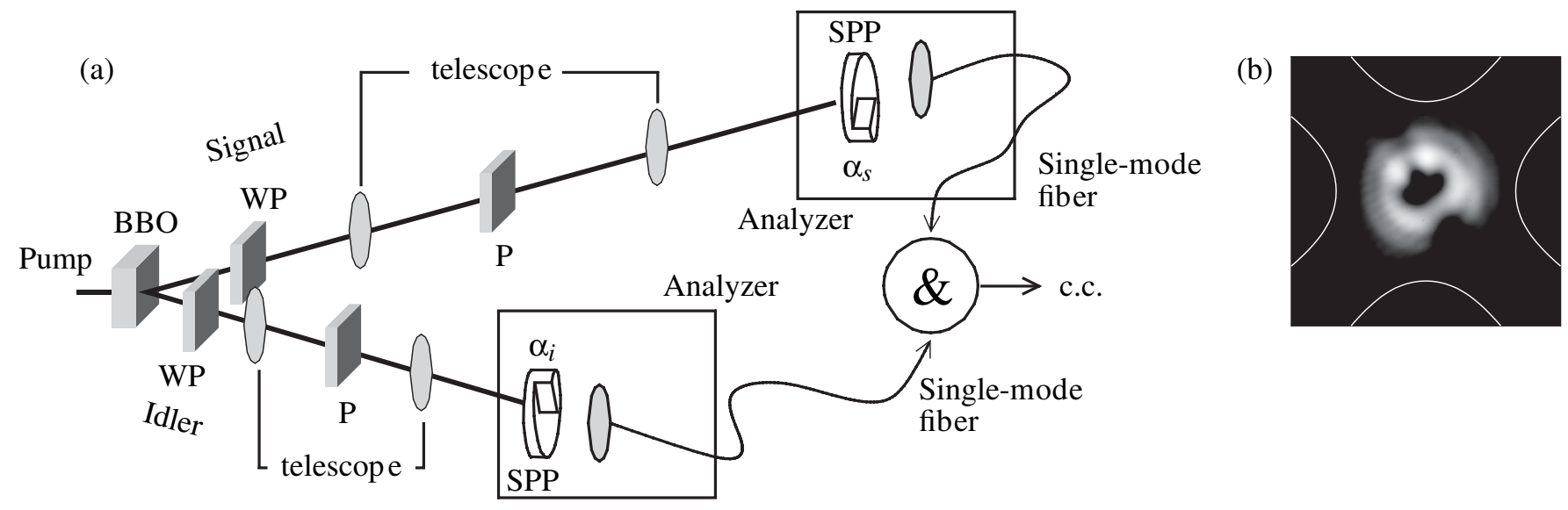

FIG. 3. (a) The SPDC setup for establishing high-dimensional entanglement with two analyzers. The SPDC crystal (BBO) is tilted so that the angle between the signal and idler arms is $6^{\circ}$. In both arms, one encounters a 0.5 mm-thick wave plate (WP) for walk-off compensation, a telescope that images the nonlinear crystal onto the SPP, a Polarcor ${ }^{\mathrm{TM}}$ polarizer $(\mathrm{P})$ and a red filter (not shown) to eliminate stray pump light. The down-converted photons are focused with microscope objectives onto single-mode fibers leading to single-photon-counting modules; coincidence counts are measured. Both SPPs can be rotated ( $\alpha_{s}$ and $\left.\alpha_{i}\right)$ around the respective beam axes. (b) An experimental far-field intensity pattern of the analyzer mode. The white curves indicate the $90 \%$ isointensity lines of the SPDC rings, illustrating that the analyzer mode completely falls in the region of twin-photon degeneracy.

shifts accordingly. Consequently, we find, according to the quantum-mechanical prediction, that the parabolic coincidence fringe depends only on the relative orientation of the SPPs. Analytical calculations in which one assumes a completely separable (as opposed to entangled) twophoton state result in fringes of zero visibility. In these calculations it is assumed, following literature [3,5] for similar experimental configurations, that OAM is conserved per pair in the SPDC process. Therefore, in the context of a quantum-mechanical description of the SPDC process, the experimental data show entanglement.

Based on the present set of data we cannot prove quantum nonlocality by using the two-dimensional ClauserHorne-Shimony-Holt version of the Bell inequality [14], in contrast to what we predicted earlier [8]. Notwithstanding the fact that the orhogonal half-integer $\ell$ states with $\alpha=0$ and $\alpha=\pi$ may be used to span a twodimensional subspace [8], when continuously varying $\alpha$ we probe a space of much larger dimensionality. Ignoring this, as in Ref. [8], invalidates the dichotomy required by the fair-sampling assumption [15] in the smaller, 2D subspace and leads to an invalid Bell parameter. Instead, as alluded to [8], a generalized Bell inequality has to be used $[16,17]$.

The dimensionality of the Hilbert space in which each photon of the pair lives must be $D>2$ as can be concluded from the parabolic shape of the fringes in Fig. 4; after all, for $D=2$, as in polarization entanglement, a sinusoidal fringe would appear.

To estimate the maximum dimensionality $D$ of the subspace probed in the experiment, we determine the number of entangled modes emitted by the BBO crystal, expressed by the Schmidt number $K[18,19]$. Here, we follow the Law-Eberly approximate analytical theory [6],

$$
K>\frac{1}{4}\left(\sqrt{\frac{L}{w_{0}^{2} k_{p}}}+\sqrt{\frac{w_{0}^{2} k_{p}}{L}}\right)^{2} .
$$

Our experimental values for the pump-beam waist $w_{0}$, crystal length $L$, and the pump wave number $k_{p}$ result in a lower limit to the Schmidt number, $K>3700 \pm 100$. A comparison between the approximate and exact theories for the value of $K$ shows that, for high values of $K$, as in the present case, the approximate theory yields a severe underestimation.

To make sure that our analyzers are not limiting the dimensionality of the probed subspace, we need to establish that their modal bandwidth is sufficiently large, i.e., $\gg$ 3700 . This can be accomplished by examining the conversion efficiency of the two analyzers, positioned as an optical train in a classical-optics experiment: we use one analyzer to convert the fundamental Gaussian mode from a laser beam to an extended superposition of LG modes, while the other is used to convert this superposition back to the fundamental Gaussian mode. The conversion efficiency, which we found to be $98 \%$, basically tells us how well the modal spectra of the two analyzers overlap. Combined with knowledge of the modal spectrum of a single analyzer (cf. Fig. 2), this value yields the modal bandwidth $N$ of the analyzers. The experimental value of 98\% [11], which is the fidelity of one analyzer mode as measured by the other analyzer, corresponds to a modal bandwidth $N \approx 125000$ (which roughly corresponds, in fact, to the diffraction limit of the full optical setup). Consequently, the analyzers are not a bottleneck in this experiment and allow us to probe entanglement with the full dimensionality as emitted by the crystal. 

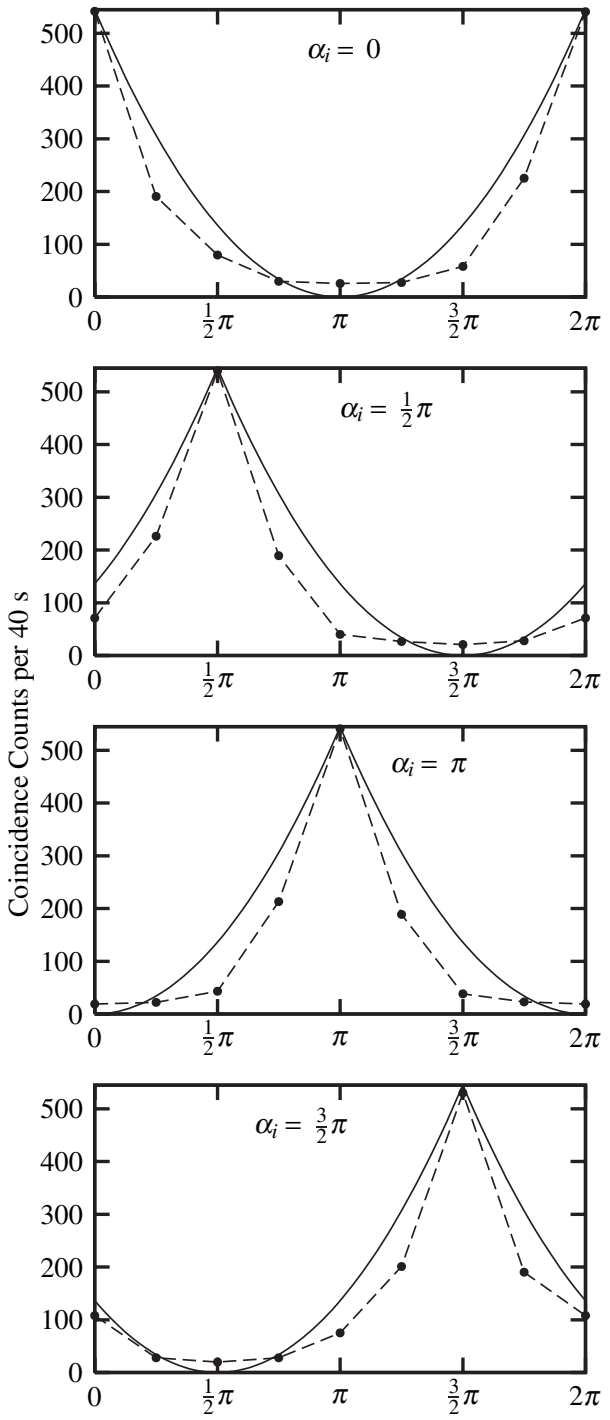

$\alpha_{s}$

FIG. 4. The measured coincidence fringes (circles and broken line segments to guide the eye) and the theoretical fringes (solid curves) as a function of the orientation $\alpha_{s}$ of the SPP in the signal arm. The parabolic fringe shifts concordantly with the orientation $\alpha_{i}$ of the SPP in the idler arm.

In conclusion, we have reported an experiment on SPDC twin photons demonstrating, within the quantummechanical description of SPDC, the entanglement of twin photons with respect to their fractional OAM. As this experiment involves a unitary transformation of basis from the natural LG states with integer OAM to a new basis with fractional OAM, the data explicitly shows that a very large part of the spatial Hilbert space is entangled; we estimate the dimensionality of the generated twin photons to be $D>3700$ per photon. The entangled space that we have probed in this experiment only represents one particular cross section of the full Hilbert space of (spatial) two-photon states. Presently, we are designing analyzers with alternative spatial geometries and step heights, and, additionally, analyzers that operate on both amplitude and phase of the photon state. Experiments with such analyzers will possibly allow us to sample a large variety of cross sections of the full Hilbert space.

We acknowledge J. A. W. van Houwelingen for his work on testing the spiral phase plates. This work is part of the research program of the "Stichting voor Fundamenteel Onderzoek der Materie (FOM)" and is supported by the EU program ATESIT.

*Also at cosine Science \& Computing, Niels Bohrweg 11, 2333 CA Leiden, The Netherlands.

${ }^{\dagger}$ Also at Philips Research Laboratories, Prof. Holstlaan 4, 5656 AA Eindhoven, The Netherlands.

[1] L. Allen, M. W. Beijersbergen, R. J. C. Spreeuw, and J. P. Woerdman, Phys. Rev. A 45, 8185 (1992).

[2] S. J. van Enk and G. Nienhuis, Opt. Commun. 94, 147 (1992).

[3] S. Franke-Arnold, S. M. Barnett, M. J. Padgett, and L. Allen, Phys. Rev. A 65, 033823 (2002).

[4] S.P. Walborn, A. N. de Oliveira, R. S. Thebaldi, and C. H. Monken, Phys. Rev. A 69, 023811 (2004).

[5] A. Mair, A. Vaziri, G. Weihs, and A. Zeilinger, Nature (London) 412, 313 (2001).

[6] C. K. Law and J.H. Eberly, Phys. Rev. Lett. 92, 127903 (2004).

[7] M. N. O'Sullivan-Hale, I. A. Khan, R. W. Boyd, and J. C. Howell, Phys. Rev. Lett. 94, 220501 (2005).

[8] S. S. R. Oemrawsingh, A. Aiello, E. R. Eliel, G. Nienhuis, and J. P. Woerdman, Phys. Rev. Lett. 92, 217901 (2004).

[9] M. W. Beijersbergen, R. P. C. Coerwinkel, M. Kristensen, and J. P. Woerdman, Opt. Commun. 112, 321 (1994).

[10] S. S. R. Oemrawsingh, J. A. W. van Houwelingen, E. R. Eliel, J.P. Woerdman, E. J. K. Verstegen, J. G. Kloosterboer, and G. W. 't Hooft, Appl. Opt. 43, 688 (2004).

[11] S. S. R. Oemrawsingh, E. R. Eliel, J. P. Woerdman, E. J. K. Verstegen, J. G. Kloosterboer, and G. W. 't Hooft, J. Opt. A Pure Appl. Opt. 6, S288 (2004).

[12] This was established with a Zygo interferometer.

[13] P. G. Kwiat, K. Mattle, H. Weinfurter, A. Zeilinger, A. V. Sergienko, and Y. Shih, Phys. Rev. Lett. 75, 4337 (1995).

[14] J. F. Clauser, M. A. Horne, A. Shimony, and R. A. Holt, Phys. Rev. Lett. 23, 880 (1969).

[15] A. Garuccio and V. A. Rapisarda, Nuovo Cimento Soc. Ital. Fis. 65A, 269 (1981).

[16] D. Collins, N. Gisin, N. Linden, S. Massar, and S. Popescu, Phys. Rev. Lett. 88, 040404 (2002).

[17] A. Vaziri, G. Weihs, and A. Zeilinger, Phys. Rev. Lett. 89, 240401 (2002).

[18] C. K. Law, I. A. Walmsley, and J.H. Eberly, Phys. Rev. Lett. 84, 5304 (2000).

[19] J. P. Torres, A. Alexandrescu, and L. Torner, Phys. Rev. A 68, 050301 (2003). 